\title{
Fuzzy Relational Mathematical Morphology: Erosion and Dilation
}

\author{
Alexander Šstak ${ }^{1,2(\bowtie)}$, Ingrīda Uljane ${ }^{1,2(\bowtie)}$, and Patrik Eklund ${ }^{3}$ \\ 1 Institute of Mathematics, CS University of Latvia, Riga 1459, Latvia \\ aleksandrs.sostaks@lumii.lv \\ 2 Department of Mathematics, University of Latvia, Riga 1004, Latvia \\ ingrida.uljane@lu. lv \\ 3 Department of Computing Science, Umeå University, 90187 Umeå, Sweden \\ peklund@cs.umu.se
}

\begin{abstract}
In the recent years, the subject if fuzzy mathematical morphology entered the field of interest of many researchers. In our recent paper [23], we have developed the basis of the (unstructured) $L$-fuzzy relation mathematical morphology where $L$ is a quantale. In this paper we extend it to the structured case. We introduce structured $L$-fuzzy relational erosion and dilation operators, study their basic properties, show that under some conditions these operators are dual and form an adjunction pair. Basing on the topological interpretation of these operators, we introduce the category of $L$-fuzzy relational morphological spaces and their continuous transformations.
\end{abstract}

Keywords: $L$-fuzzy relational erosion $\cdot L$-fuzzy relational dilation • $L$-fuzzy relational morphological spaces $\cdot$ Duality $\cdot$ Adjointness · Continuous transformations

\section{Introduction}

Mathematical morphology has its origins in geological problems centered in the processes of erosion and dilation. The founders of mathematical morphology are engineer G. Matheron [18] and his student, engineer J. Serra [22]. The idea of the classical mathematical morphology can be explained as the process of modifying a subset $A$ of a cube in an n-dimensional Euclidean space $\mathbb{R}^{n}$ by cutting out pieces of $B$ from $A$ (in case of erosion) or glueing them down to the set $A$ (in case of dilation). The set $B$, intuitively, small if compared with $A$, is called the structuring set. In the first works on fuzzy morphology $A$ and $B$ were crisp sets, however soon the interest of some researchers was directed also to the case when $A$ and $B$ could be fuzzy. This allowed to describe gray scale processes of erosion and dilation. The first fundamental works on fuzzy mathematical morphology are the two papers by B. De Baets, E. Kerre and M. Gupta [7], [8].

The first and the second authors are thankful for the partial financial support from the project No. Lzp-2018/2-0338.

(c) Springer Nature Switzerland AG 2020

M.-J. Lesot et al. (Eds.): IPMU 2020, CCIS 1239, pp. 712-725, 2020.

https://doi.org/10.1007/978-3-030-50153-2_52 
In the first period of the development of fuzzy mathematical morphology the domain where the operators of erosion and dilation were defined was restricted by Euclidean spaces $\mathbb{R}^{n}$, both in crisp and fuzzy approaches. This framework was usually adequate for studying practical problems in geology, it was appropriate also for applications of mathematical morphology in different applied sciences, in particular in pattern recognition, image processing, digital topology, etc. Additionally it was convenient since all basic constructions of fuzzy morphology, in particular, "cutting" and "glueing" pieces $B$ from or to $A$ were defined by means of the use of the linear structure in the Euclidean space. However, some researchers were attracted by the idea to extend basic concepts and constructions of fuzzy morphology from $\mathbb{R}^{n}$ to a more general context. This idea was interesting not only theoretically, but also in view of possible applications in some tasks beyond the classical ones. The principle how to find the "correct" extensions for the definitions were found in the interrelations between erosion and dilation operators that can be observed in almost all "classical" approaches. Namely, the principal features are that erosion $\varepsilon$ and dilation $\delta$ are dual operators and the pair $(\varepsilon, \delta)$ is an adjunction.

This observation has lead to two mainstreams in the generalized approach to fuzzy mathematical morphology: the algebraic and the relational one. The algebraic approach, in its most general form, is based on two complete lattices $L_{1}$ and $L_{2}$ and two mappings: a mapping $\varepsilon: L_{1} \rightarrow L_{2}$ that preserves arbitrary infima, and a mapping $\delta: L_{2} \rightarrow L_{1}$ that preserves arbitrary joins. These mappings $\varepsilon$ and $\delta$ should be related by Galois connection. This approach was initiated by Heijecman [12] and further developed by I. Bloch [3], [4] and some other authors. The second, less formal relational approach, considers a set $X$ equipped with some relation $R$; this relation is used instead of linear transformations applied in the classical case, that is when $X$ is the Euclidean space, see e.g. [19], see also [23].

In this paper, we start to develop an approach to $L$-fuzzy relational morphology in which, as different from e.g. [19] and [23], the erosion and dilation are structured by some $L$-fuzzy set $B$.

The paper consists of five sections. In the first one we recall and specify terminology related to quantales and $L$-fuzzy relations. In the second section images and preimages of $L$-fuzzy sets under $L$-fuzzy relations are considered; these images and preimages are closely related to the operators of erosion and dilation introduced and studied in Sect.3. In the fourth section we consider interrelations between operators of erosion and dilation. Namely, we show that under some conditions they are dual and make an adjoint pair. In the next, fifth section, we introduce the category $\mathbb{M}(L)$ of $L$-fuzzy relational morphological spaces and its subcategory $\mathbb{M}^{p}(L)$ of unstructured fuzzy relational morphological spaces. We study some properties of this categories and compare these categories with certain categories of Fuzzy Topology. In the last, Conclusion section, we list some directions where our approach to the concept of fuzzy morphological spaces could be further developed. 


\section{Preliminaries}

In this section, we recall some well-known concepts that will make the context of our work. The restricted volume of the paper does not allow to reproduce here all information used in the work. A reader is referred to the monographs $[11,21]$ and other standard references sources for the remaining details.

\subsection{Lattices, Quantales, Girard Monoids and MV-algebras}

In our paper, $(L, \leq, \wedge, \vee)$ is a complete infinitely distributive lattice with bottom and top elements $0_{L}$ and $1_{L}$ respectively. Given a binary associative monotone operation $*: L \times L \rightarrow L$ on $(L, \leq, \wedge, \vee)$, the tuple $(L, \leq, \wedge, \vee, *)$ is called a quantale if $*$ commutes over arbitrary joins:

$$
\alpha *\left(\bigvee_{i \in I} \beta_{i}\right)=\bigvee_{i \in I}\left(\alpha * \beta_{i}\right) \quad \forall \alpha \in L, \forall\left\{\beta_{i} \mid i \in I\right\} \subseteq L
$$

The operation $*$ will be referred to as the product or the conjunction. A quantale is called commutative if the product $*$ is commutative. A quantale is called integral if the top element $1_{L}$ acts as the unit, that is $1_{L} * \alpha=\alpha$ for every $\alpha \in L$.

In a quantale a further binary operation $\mapsto: L \times L \rightarrow L$, the residuum, can be introduced as associated with operation $*$ of the quantale $(L, \leq, \wedge, \vee, *)$ via the Galois connection, that is $\alpha * \beta \leq \gamma \Longleftrightarrow \alpha \leq \beta \mapsto \gamma$ for all $\alpha, \beta, \gamma \in L$. Explicitly residium can be defined by $\alpha \mapsto \beta=\bigvee\{\lambda \in L \mid \lambda * \alpha \leq \beta\}$.

Further, a unary operator ${ }^{c}: L \rightarrow L$ is called negation if it is an order reversing involution, that is $\alpha \leq \beta \Longrightarrow \beta^{c} \leq \alpha^{c}$ and $\left(\alpha^{c}\right)^{c}=\alpha$ for all $\alpha, \beta \in L$. For us it is important that negation ${ }^{c}$ in a quantale is well-coordinated with the original quantale structure $(L, \leq, \wedge, \vee, *)$. Explicitly, this means that the negation should be defined according to the laws of fuzzy logic, that is $a^{c}=a \mapsto$ 0 . Therefore, to satisfy the properties of the negation, we have to request that

$$
(\alpha \mapsto 0) \mapsto 0=\alpha \forall \alpha \in L .
$$

Quantales $(L, \leq, \wedge, \vee, *)$ satisfying this property are called Girard monoids [16] or Girard quantales. Girards quantales are a generalization of the concept of an MV-algebra, see e.g. [13], [14]: A quantale is called an MV-algebra if

$$
(\alpha \mapsto \beta) \vee \beta=\alpha \vee \beta \text { for all } \alpha, \beta \in L .
$$

In an $M V$-algebra $(L, \leq, \wedge, \vee, *)$ operation $*$ distributes also over arbitrary meets see e.g. [13], [14]:

$$
\alpha *\left(\bigwedge_{i \in I} \beta_{i}\right)=\bigwedge_{i \in I}\left(\alpha * \beta_{i}\right) \forall \alpha \in L, \forall\left\{\beta_{i} \mid i \in I\right\} \subseteq L .
$$

In a Girard quantale $(L, \leq, \wedge, \vee, *)$ a further binary operation $\oplus$, so called coproduct or disjunction, can be defined by setting

$$
\alpha \oplus \beta=\left(\alpha^{c} * \beta^{c}\right)^{c} \forall \alpha, \beta \in L .
$$


Co-product is a commutative associative monotone operation and, in case $(L, \leq$, $\wedge, \vee, *)$ iks integral, $0_{L}$ acts as a zero, that is $\alpha \oplus 0_{L}=\alpha$ for every $\alpha \in L$. Important properties of operations in Girard quantales are given in the next Lemma:

Lemma 1. Operation $\oplus$ in a Girard quantale $\left(L, \leq_{L}, \wedge_{L}, \vee_{L}, *\right)$ is distributive over arbitrary meets:

$$
\alpha \oplus\left(\bigwedge_{i \in I} \beta_{i}\right)=\bigwedge_{i \in I}\left(\alpha \oplus \beta_{i}\right) \quad \forall \alpha \in L, \forall\left\{\beta_{i} \mid i \in I\right\} \subseteq L .
$$

The proof follows from the following series of equalities justified by definitions: $\alpha \oplus\left(\bigwedge_{i \in I} \beta_{i}\right)=\left(\alpha^{c} *\left(\bigwedge_{i \in I} \beta_{i}\right)^{c}\right)^{c}=\left(\alpha^{c} *\left(\bigvee_{i \in I} \beta_{i}^{c}\right)\right)^{c}=\left(\bigvee_{i \in I}\left(\alpha^{c} * \beta_{i}^{c}\right)\right)^{c}=$ $\bigwedge_{i \in I}\left(\left(\left(\alpha^{c}\right)^{c} \oplus\left(\beta_{i}^{c}\right)^{c}\right)^{c}\right)^{c}=\bigwedge_{i \in I}\left(\alpha \oplus \beta_{i}\right)$.

In a similar way one can prove the following Lemma.

Lemma 2. If in a Girard quantale $(L, \leq, \wedge, \vee, *)$ operation * distributes over arbitrary meets, then the corresponding operation $\oplus$ distributes over arbitrary joins:

$$
\alpha \oplus\left(\bigvee_{i \in I} \beta_{i}\right)=\bigvee_{i \in I}\left(\alpha \oplus \beta_{i}\right) \quad \forall \alpha \in L, \forall\left\{\beta_{i}: i \in I\right\} \subseteq L
$$

We will need also the following Lemma, the proof of which can be found in $[13$, Lemme 1.4]; we reformulate it a way convenient for our use:

Lemma 3. In a Girard quantale $(L, \leq, \wedge, \vee, *)$ the following equality holds:

$$
\alpha \mapsto \beta=(\alpha *(\beta \mapsto 0)) \mapsto 0 \quad \forall \alpha, \beta \in L
$$

A quantale $(L, \leq, \wedge, \vee, *)$ is called divisible if

$$
a \leq b \Longleftrightarrow \text { exists } d \in L \text { such that } a * d=b
$$

see e.g. [15, p. 128]. It is known that every MV-algebra is divisible [15, p. 129]. We will need a stronger version of this property:

Definition 1. A quantale is called strongly divisible if

$$
a * \lambda=b * c \Longleftrightarrow \exists \mu \in L \text { such that } a * c * \mu=b \quad \forall a, b, c, \lambda \in L .
$$

Lemma 4. In a strongly divisible quantale $L$ the equality $a \mapsto b * c=(a \mapsto b) * c$ holds for all $a, b, c \in L$.

Proof. $(a \mapsto b) * c=c * \bigvee\{\lambda \mid \lambda * a \leq b\}=\bigvee\{\lambda * c \mid \lambda * a \leq b\} \leq \bigvee\{\lambda * c \mid$ $\lambda * a * c \leq b * c\} \leq \bigvee\{\mu \mid \mu \leq b * c\}=a \mapsto b * c$

On the other hand, by strong divisibility $a \mapsto b * c=\bigvee\{\lambda \in L \mid \lambda * a \leq b\} \leq$ $\bigvee\{\mu \in L \mid \mu * a * c \leq b\}=c * \bigvee\{\mu \in L \mid \mu * a \leq b\}=c *(a \mapsto b)$

In our work $(L, \leq, \wedge, \vee, *)$ is always an integral commutative quantale, sometimes satisfying additional, explicitly stated, conditions. 


\section{$2.2 \quad L$-fuzzy Relations, $L$-fuzzy Relational Sets}

Definition 2 (see e.g. [25]). An L-fuzzy relation from a set $X$ to a set $Y$ is an $L$-fuzzy subset of the product $X \times Y$, that is a mapping $R: X \times Y \rightarrow L$. In case when $X=Y$ it is called an L-fuzzy relation on the set $X$. The triple $(X, Y, R)$ where $R$ is an L-fuzzy relation from $X$ to $Y$ is called an $L$-fuzzy relational triple and if $X=Y$ the pair $(X, R)$ is called an $L$-fuzzy relational set.

We will need some special properties of $L$-fuzzy relations specified below:

Definition 3 (see e.g. [24]). An L-fuzzy relation $R: X \times Y \rightarrow L$ is called left connected if $\bigwedge_{y \in Y} \bigvee_{x \in X} R(x, y)=1_{L} . R$ is called strongly left connected if for every $y \in Y$ there exists $x \in X$ such that $R(x, y)=1_{L}$. An $L$-fuzzy relation $R$ is called right connected if $\bigwedge_{x \in X} \bigvee_{y \in Y} R(x, y)=1_{L} \cdot R$ is called strongly right connected if for every $x \in X$ there exists $y \in Y$ such that $R(x, y)=1_{L}$. An $L$ - fuzzy relation $R$ on a set $X$ is called reflexive if $R(x, x)=1_{L}$ for every $x \in X$. An L-fuzzy relation $R$ on a set $X$ is called symmetric, if $R(x, y)=$ $R(y, x)$ for all $x, y \in X$. An L-fuzzy relation $R$ on a set $X$ is called transitive if $R(x, y) * R(y, z) \leq R(x, z)$ for all $x, y, z \in X$.

\section{$3 \quad$ Image and Preimage Operators on $L$-fuzzy Power-Sets Induced by $L$-fuzzy Relations}

The subject of this section is, what we call, upper and lower image and preimage operators induced by an $L$-fuzzy relation $R: X \times Y \rightarrow L$. TAs we will see they are closely related to the operators of fuzzy relational erosion and dilation. These operators $R^{\rightarrow}: L^{X} \rightarrow L^{Y}$, and their basic properties can be found in different papers where $L$-fuzzy power-sets are involved. For reader's convenience we briefly discuss them here.

Definition 4. The upper image of an $L$-fuzzy set $A \in L^{X}$ under L-fuzzy relation $R: X \times Y \rightarrow L$ is the $L$-fuzzy set $R^{\rightarrow}(A) \in L^{Y}$ defined by $R^{\rightarrow}(A)(y)=$ $\bigvee_{x \in X} R(x, y) * A(x)$ for all $A \in L^{X}, y \in Y$.

Definition 5. The upper preimage of an $L$-fuzzy set $A \in L^{Y}$ under $L$-fuzzy relation $R: X \times Y \rightarrow L$ is the $L$-fuzzy set $R^{\leftarrow}(A) \in L^{X}$ defined by $R^{\leftarrow}(A)(x)=$ $\bigvee_{y \in Y} R(x, y) * A(y)$ for all $A \in L^{Y}, x \in X$.

Definition 6. The lower image of an $L$-fuzzy set $A \in L^{X}$ under L-fuzzy relation $R: X \times Y \rightarrow L$ is the L-fuzzy set $R^{\Rightarrow}(A) \in L^{Y}$ defined by $R^{\Rightarrow}(A)(y)=$ $\bigwedge_{x \in X}(R(x, y) \mapsto A(x))$ for all $A \in L^{X}, y \in Y$.

Definition 7. The lower preimage of an $L$-fuzzy set $A \in L^{Y}$ under $L$-fuzzy relation $R: X \times Y \rightarrow L$ is the L-fuzzy set $R^{\Leftarrow}(A) \in L^{X}$ defined by $R^{\Leftarrow}(A)(x)=$ $\bigwedge_{y \in Y}(R(x, y) \mapsto A(y))$ for all $A \in L^{Y}, y \in Y$.

Proposition 1. If relation $R: X \times Y \rightarrow L$ is strongly left connected, then $R^{\Rightarrow}(A) \leq R^{\rightarrow}(A)$ for every $A \in L^{X}$. 
Proof. Given $y \in Y$, we take $x_{y} \in X$ such that $R\left(x_{y}, y\right)=1_{L}$. Then $\bigwedge_{x \in X} R(x, y) \mapsto A(x) \leq R\left(x_{y}, y\right) \mapsto A\left(x_{y}\right)=1_{L} \mapsto A\left(x_{y}\right)=A\left(x_{y}\right)$.

On the other hand, $\bigvee_{x \in X} R(x, y) * A(x) \geq R\left(x_{y}, y\right) * A\left(x_{y}\right)=A\left(x_{y}\right)$.

Proposition 2. If relation $R: X \times Y \rightarrow L$ is strongly right connected, then $R^{\leftarrow}(A) \leq R^{\leftarrow}(A)$ for every $A \in L^{Y}$.

Proof. Let $x \in X$ be fixed and let $y_{x} \in X$ satisfy $R\left(x, y_{x}\right)=1_{L}$. Then $R^{\leftarrow}(A)(x)=\bigvee_{y}(R(x, y) * A(y)) \geq R\left(x, y_{x}\right) * A\left(y_{x}\right)=A\left(y_{x}\right)$. In its turn, $R^{\Leftarrow}(A)(x)=\bigwedge_{y}(R(x, y) \mapsto A(y)) \leq R\left(x, y_{x}\right) \rightarrow A\left(y_{x}\right)=A\left(y_{x}\right)$.

\section{Operators of Structured $L$-fuzzy Relational Erosion and Dilation}

\subsection{Structured Relational $L$-fuzzy Erosion}

Modifying the definition of $L$-fuzzy relational erosion given in [19], see also [23] to the case when the fuzzy erosion of a fuzzy set $A \in L^{X}$ is structured by a fuzzy set $B \in L^{Y}$, we come to the following definition

Definition 8. Given $A \in L^{X}$ and $B \in L^{Y}$, the erosion of $A$ structured by $B$ in a fuzzy relational triple $(X, Y, R)$ is the L-fuzzy set $\varepsilon_{R}(A, B) \in L^{Y}$ defined by

$$
\varepsilon_{R}(A, B)(y)=\left(\bigwedge_{x \in X}(R(x, y) \mapsto A(x))\right) * B^{c}(y)
$$

Considering erosion for all $A \in L^{X}$ when $B \in L^{Y}$ is fixed, we get the operator of erosion $\varepsilon_{R}(\cdot, B): L^{X} \rightarrow L^{Y}$.

Thus $L$-fuzzy erosion $\varepsilon_{R}\left(\cdot, 0_{X}\right): L^{X} \rightarrow L^{Y}$ is actually the lower image operator $R \Rightarrow$ induced by $L$-fuzzy relation $R: X \times Y \rightarrow L$.

In the next proposition we collect some properties of erosion operators.

Proposition 3. (1) $\varepsilon_{R}\left(1_{X}, B\right)=B^{c}$. If $R$ is left connected, then $\varepsilon_{R}\left(a_{X}, B\right)=$ $a * B^{c}$ for every $a \in L$ where $a_{X}: X \rightarrow L$ is the constant function with value $a \in L$.

(2) Operator $\varepsilon_{R}(\cdot, B): L^{X} \rightarrow L^{Y}$ is non-decreasing, that is if $A_{1} \leq A_{2} \in L^{X}$ then $\varepsilon_{R}\left(A_{1}, B\right) \leq \varepsilon_{R}\left(A_{2}, B\right)$.

(3) If $B_{1} \leq B_{2} \in L^{\bar{Y}}$ then for every $A \in L^{X} \varepsilon_{R}\left(A, B_{1}\right) \geq \varepsilon_{R}\left(A, B_{2}\right)$.

(4) If $*$ is distribute over arbitrary meets, then, given a family $\left\{A_{i} \mid i \in I\right\} \subseteq L^{X}$ and $B \in L^{Y}$, we have $\varepsilon_{R}\left(\bigwedge_{i \in I} A_{i}, B\right)=\bigwedge_{i \in I} \varepsilon_{R}\left(A_{i}, B\right)$.

Proof. (1) From the definition it is clear that for every $y \in Y$ we have $\varepsilon_{R}\left(a_{X}, B\right)(y)=\left(\bigwedge_{x \in X}(R(x, y) \mapsto a) * B^{c}(y)=\left(\left(\bigvee_{x \in X} R(x, y)\right) \mapsto a\right) * B^{c}(y)\right.$. In case $a=1_{L}$, we have $\varepsilon_{R}\left(1_{X}, B\right)(y)=B^{c}(y)$ for every $y \in Y$. In its turn, if $R(x, y)$ is left connected, then $\bigvee_{x \in X} R(x, y)=1_{L}$ for every $y \in Y$ and hence $\varepsilon_{R}\left(a_{X}, B\right)(y)=\left(1_{L} \mapsto a\right) * B^{c}(y)=a_{Y} * B^{c}(y)$.

The statements (2) and (3) are obvious. 
(4) Given a family of $L$-fuzzy sets $\left\{A_{i} \mid i \in I\right\}$ and $y \in$ $Y$, by meet-distributivity of $*$ we have $\varepsilon_{R}\left(\bigwedge_{i \in I} A_{i}, B\right)(y)=\bigwedge_{x \in X}$ $\left(\bigwedge_{i \in I}\left(R(x, y) \mapsto A_{i}(x)\right)\right) * B^{c}(y)=\bigwedge_{i \in I}\left(\bigwedge_{x \in X}\left(R(x, y) \mapsto A_{i}(x)\right) * B^{c}(y)\right)=$ $\bigwedge_{i \in I} \varepsilon_{R}\left(A_{i}, B\right)(y)$.

In the rest of this subsection, we consider the case when $X=Y$, that is when $R: X \times Y \rightarrow L$ is an $L$-fuzzy relation on a set $X$. In this case erosion has some important additional properties.

Proposition 4. If $L$-fuzzy relation is reflexive, then for every $A \in L^{X}$ and every $B \in L^{X}$ it holds $\varepsilon_{R}(A, B) \leq A * B^{c}$. In particular, $\varepsilon_{R}(A, A) \leq A * A^{c}$.

Proof Notice that for every $y \in Y$

$\varepsilon_{R}(A, B)(y)=\left(\bigwedge_{x \in X}(R(x, y) \mapsto A(x))\right) * B^{c}(y) \leq(R(y, y) \mapsto A(y)) * B^{c}(y)=$ $\left(1_{L} \mapsto A(y)\right) * B^{c}(y)=A(y) * B^{c}(y)=\left(A * B^{c}\right)(y)$.

Corollary 1. If $L$-fuzzy relation $R$ is reflexive, then $\varepsilon_{R}(A, B) \leq A$.

To formulate the next proposition, we denote $\bar{B}=\sup \{B(y): y \in Y\}$.

Proposition 5. If $L$-fuzzy relation $R$ is reflexive and symmetric and $*$ distributes over arbitrary meets, then for any L-fuzzy sets $A \in L^{X}$ and $B \in L^{Y}$ it holds

$$
\varepsilon_{R}(A, B) *(\bar{B})^{c} \leq \varepsilon_{R}\left(\varepsilon_{R}(A, B), B\right) \leq \varepsilon_{R}(A, B) .
$$

Proof. Inequality $\varepsilon_{R}\left(\varepsilon_{R}(A, B), B\right) \leq \varepsilon_{R}(A, B)$ follows from Corrolary 1. To show the other inequality let $X=Y=Z$ be sets, $R: X \times Y \rightarrow L$ be an $L$-fuzzy relation, define $R: Y \times Z \rightarrow L$ in the same way as the given $L$-fuzzy relation $R: X \times Y \rightarrow L$ and let $z \in Z$. Then by meet-distributivity of $*$ and symmetry of $R$ and twice applying inequality $a \mapsto b * c \leq(a \mapsto b) * c$ we have

$\varepsilon_{R}\left(\varepsilon_{R}(A, B), B\right)(z)=\left[\bigwedge_{y \in Y}\left(R(y, z) \mapsto \varepsilon_{R}(A, B)(y)\right)\right] * B^{c}(z)=$

$\left[\bigwedge_{y \in Y}\left(R(y, z) \mapsto\left(\bigwedge_{x \in X}(R(x, y) \mapsto A(x))\right) * B^{c}(y)\right)\right] * B^{c}(z) \geq$

$\left.\bigwedge_{y \in Y}\left(R(y, z) \mapsto(R(z, y) \mapsto A(z)) * B^{c}(y)\right)\right] * B^{c}(z) \geq$

$\left[\bigwedge_{y \in Y}\left(R(y, z) \mapsto\left(R(z, y) \mapsto A(z) * B^{c}(y)\right)\right] * B^{c}(z) \geq\right.$

$\bigwedge_{y \in Y}\left(\left(R(y, z) \mapsto A(z) * B^{c}(y)\right)\right] * B^{c}(z) \geq$

$\bigwedge_{y \in Y}\left((R(y, z) \mapsto A(z)) * B^{c}(z) * B^{c}(y)\right) \geq$

$\bigwedge_{y \in Y}(R(y, z) \mapsto A(z)) * B^{c}(z) *(\bar{B})^{c}=\varepsilon_{R}(A, B)(z) *(\bar{B})^{c}$.

In case $B=0_{Y}$ we do not need to use meet-distributivity of $*$ and so we have:

Corollary 2. If the $L$-fuzzy relation $R$ is reflexive and symmetric and $B=0_{Y}$, then for any $A \in L^{X}$ it holds $\varepsilon_{R}\left(\varepsilon_{R}\left(A, 0_{Y}\right), 0_{Y}\right)=\varepsilon_{R}\left(A, 0_{Y}\right)$. In particular this means that operator $\varepsilon_{R}\left(\cdot, 0_{X}\right): L^{X} \rightarrow L^{X}$ is idempotent. 


\subsection{Structured $L$-fuzzy Dilation}

As before, let $X, Y$ be sets, $R: X \times Y \rightarrow L$ an $L$-fuzzy relation and let $A \in L^{X}$ and $B \in L^{Y}$ be $L$-fuzzy sets. Generalizing definition of relational dilation of the $L$-fuzzy set given in [19], see also [23], for the situation when dilation of $A$ is structured by $B$, we come to the following definition:

Definition 9. Given $A \in L^{X}$, its $L$-fuzzy dilation structured by $B \in L^{Y}$ is an $L$-fuzzy set $\delta_{R}(A, B) \in L^{Y}$ defined by

$$
\delta_{R}(A, B)(y)=\left(\bigvee_{x \in X} R(y, x) * A(x)\right) \oplus B(y)
$$

Considering dilation for all $A \in L^{X}$ when the structuring L-fuzzy set $B$ is fixed, we get the operator of dilation $\delta_{R}(\cdot, B): L^{X} \rightarrow L^{Y}$.

In the next proposition we collect basic properties of dilation operator $\delta_{R}(\cdot, B)$.

Proposition 6. Let $R: X \times Y \rightarrow L$ be an L-fuzzy relation. Then

(1) $\delta_{R}\left(0_{X}, B\right)=0_{Y}$ and if $R$ is right connected, then $\delta_{R}\left(a_{X}, B\right)=a_{Y} \oplus B$ for any $a \in L$, and in particular $\delta_{R}\left(1_{X}, B\right)=1_{Y}$.

(2) $A_{1} \leq A_{2} \in L^{X} \Longrightarrow \delta_{R}\left(A_{1}, B\right) \leq \delta_{R}\left(A_{2}, B\right)$.

(3) If $B_{1} \leq B_{2} \in L^{Y}$ then for every $A \in L^{X} \quad \delta_{R}\left(A, B_{1}\right) \leq \delta_{R}\left(A, B_{2}\right)$.

(4) If operation $*$ is distributes over arbitrary joins, then given a family of $L$-fuzzy sets $\left\{A_{i} \mid i \in I\right\} \subseteq L^{X}$, it holds $\delta_{R}\left(\bigvee_{i \in I} A, B\right)=\bigvee_{i \in I} \delta_{R}\left(A_{i}, B\right)$.

Proof. (1) For every $y \in Y$ we have

$\delta_{R}\left(a_{X}, B\right)(y)=\left(\bigvee_{x \in X} R(y, x) * a_{X}(x)\right) \oplus B(y)=\left(\bigvee_{x \in X} R(y, x) * a\right) \oplus B(y)$. Hence, $\delta_{R}\left(0_{X}, B\right)(y)=0_{Y}$ for every $y \in Y$, that is $\delta_{R}\left(0_{X}, B\right)=0_{Y}$. If $R$ is right connected, then $\delta_{R}\left(a_{X}, B\right)(y)=a_{Y} \oplus B(y)$ for all $y \in Y$.

The proof of (2) and (3) is obvious.

(4) Let a family of $L$-fuzzy sets $\left\{A_{i} \mid i \in I\right\} \subseteq L^{X}$ and $y \in Y$ be given. Recalling that by Lemma 2 co-product distribures over arbitrary joins, we have $\left.\delta_{R}\left(\bigvee_{i \in I} A_{i}, B\right)\right)(y)=\left(\bigvee_{x \in X}\left(R(y, x) * \bigvee_{i \in I} A_{i}(x)\right)\right) \oplus B(y)=$ $\left(\bigvee_{x \in X}\left(\bigvee_{i \in I}\left(R(y, x) * A_{i}(x)\right)\right)\right) \oplus B(y)=$ $\left(\bigvee_{i \in I}\left(\bigvee_{x \in X}\left(R(y, x) * A_{i}(x)\right)\right)\right) \oplus B(y)=$ $\bigvee_{i \in I}\left(\bigvee_{x \in X}\left(R(y, x) * A_{i}(x)\right) \oplus B(y)\right)=\bigvee_{i \in I} \delta_{R}\left(A_{i}, B\right)(y)$

In the rest of this subsection, we consider the case when $X=Y$ that is when $R$ is an $L$-fuzzy relation on the set $X$. In this case dilation has some additional properties.

Proposition 7. If $L$-fuzzy relation $R$ is reflexive, then for every $A \in L^{X}$ and every $B \in L^{X}$ it holds $\delta_{R}(A, B) \geq A \oplus B$. In particular, $\delta_{R}(A, A) \geq A \oplus A$.

Proof. Given any point $y \in Y(=X)$, by reflexivity of $R$ we have: $\delta_{R}(A, B)(y)=$ $\left(\bigvee_{x \in X} R(y, x) * A(x)\right) \oplus B(y) \geq(R(y, y) * A(y)) \oplus B(y)=A(y) \oplus B(y)$ 
Corollary 3. If $L$-fuzzy relation $R$ is reflexive, then $\delta_{R}(A, B) \geq A$ for all $A, B \in L^{X}$.

To formulate the next proposition we recall that $\bar{B}=\sup \{B(y): y \in Y\}$

Proposition 8. If the L-fuzzy relation is reflexive and symmetric and operation * is distributes over arbitrary meets, then for all L-fuzzy sets $A \in L^{X}$ and $B \in L^{Y}$ the following inequality holds

$$
\delta_{R}(A, B) \oplus \bar{B} \geq \delta_{R}\left(\delta_{R}(A, B), B\right) \geq \delta_{R}(A, B) .
$$

Proof. The inequality $\delta_{R}\left(\delta_{R}(A, B), B\right) \geq \delta_{R}(A, B)$ follows from Corollary 3 . We establish the second inequality as follows. Let $X=Y=Z$ be sets and take some $z \in Z$. Then

$$
\begin{aligned}
& \delta_{R}\left(\delta_{R}(A, B), B\right)(z)=\left(\bigvee_{y \in Y} R(z, y) * \delta_{R}(A, B)(y)\right) \oplus B(z)= \\
& \left.\left[\bigvee_{y \in Y}\left(R(z, y) *\left(\bigvee_{x \in X} R(y, x) * A(x)\right) \oplus B(y)\right)\right)\right] \oplus B(z) \leq \\
& {\left[\bigvee_{y \in Y}(R(z, y) *(R(y, z) * A(z)) \oplus B(y))\right] \oplus B(z) \leq} \\
& {\left[\bigvee_{y \in Y}(R(z, y) *(R(y, z) * A(z)) \oplus B(y))\right] \oplus B(z)=} \\
& {\left[\bigvee_{y \in Y}(R(z, y) *(R(z, y) * A(z)) \oplus B(y))\right] \oplus B(z) \leq} \\
& \left.\left[\bigvee_{y \in Y}(R(z, y) * A(z)) \oplus B(y)\right)\right] \oplus B(z)=(\text { by Lemma2) } \\
& \left.\bigvee_{y \in Y}((R(z, y) * A(z)) \oplus B(z)) \oplus B(y)\right) \leq \\
& {\left[\bigvee_{y \in Y}(R(z, y) * A(z)) \oplus B(z)\right] \oplus \bar{B}=\delta_{R}(A, B)(y) \oplus \bar{B} .}
\end{aligned}
$$

Since in case $B=0_{Y}$ in the proof we do not need join-distributivity of the co-product $\oplus$, we get the following corollary from the previous theorem.

Corollary 4. If the L-fuzzy relation $R$ is reflexive and symmetric, then $\delta_{R}\left(\delta_{R}\left(A, 0_{Y}\right), 0_{Y}\right)=\delta_{R}\left(A, 0_{Y}\right)$ and hence operator $\delta_{R}\left(\cdot, 0_{Y}\right)$ is idempotent.

\section{Interrelations Between Fuzzy Relational Erosion and Dilation}

One of the most important attributes of mathematical morphology is the interrelations between erosion and dilation which manifest in two ways: as the adjunction between erosion and dilation and as the duality between erosion and dilation. One or both of them exist in all approaches to fuzzy morphology known to us. It is the aim of this section to study the corresponding interrelation in our case. Unfortunately, to get the analogues of these interconnections in case of structured relational erosion and dilation, we have to assume additional conditions laid down on the quantale $(L, \leq, \wedge, \vee, *)$. 


\subsection{Duality Between Fuzzy Relational Erosion and Dilation}

Theorem 1. Let $(L, \leq, \vee, \wedge, *)$ be a Girard quantale. Then for every $B \in L^{X}$ operators $\varepsilon_{R}(\cdot, B)$ and $\delta_{R}(\cdot, B)$ make a dual pair:

$$
\varepsilon_{R}^{c}(A, B)=\delta_{R}\left(A^{c}, B\right) \quad \forall A \in L^{X} .
$$

Proof. We prove the theorem by a series of equivalent transitions which are justified by the definition of Girard quantale:

$\varepsilon_{R}^{c}(A, B)(y)=$

$\left(\left(\bigwedge_{x \in X}(R(x, y) \mapsto A(x))\right) * B^{c}(y)\right) \mapsto 0=$ (definition of a Girard quntale)

$\left(\bigwedge_{x \in X}((R(x, y) \mapsto A(x)) \mapsto 0)\right) \oplus\left(B^{c}(y) \mapsto 0\right)=$ (by Lemma 3$)$

$\left(\bigvee_{x \in X} R(x, y) *(A(x) \mapsto 0)\right) \oplus B(y)=\delta_{R}\left(A^{c}, B\right)(y)$.

Corollary 5. Let $(L, \leq, \vee, \wedge, *)$ be a Girard quantale. Then for every $B \in L^{X}$

$$
\delta_{R}^{c}(A, B)=\varepsilon_{R}\left(A^{c}, B\right) \quad \forall A \in L^{X} .
$$

\subsection{Adjunction $\left(\varepsilon_{R}(\cdot, B), \delta_{R}(\cdot, B)\right)$}

When studying the problem of adjunction between operators $\varepsilon_{R}(\cdot, B)$ and $\delta_{R}(\cdot, B)$, we inevitably (?) have to assume that $*$ and $\oplus$ constitute an adjuction. In the next definition we specify what we mean by this.

Definition 10. A pair $(*, \oplus)$ is called adjunctive if for any $\alpha, \beta, \gamma \in L$

$$
\alpha \oplus \beta \leq \gamma \Longleftrightarrow \alpha \leq \gamma * \beta^{c} .
$$

Unfortunately, at the moment we have only one example of an adjunctive pair $(*, \oplus)$ - namely the one that corresponds to Łukasiewicz $t$-norm and its generalizations.

Theorem 2. Let $(L, \leq, \wedge, \vee, *)$ be a strongly divisible Girard monoid, * distribute over arbitrary meets and $(*, \oplus)$ be an adjunctive pair. Then $\left(\varepsilon_{R}(\cdot, B), \delta_{R}(\cdot, B)\right)$ is an adjunctive pair.

Proof. Since both functors $\varepsilon_{R}(\cdot, B)$ and $\delta_{R}(\cdot, B)$ are defined on the lattice $L^{X}$ and take values in the same lattice $L^{X}$, the adjunction just means that these functors are related by Galois connection, that is for any $A, C \in L^{X}$ :

$$
\delta_{R}(A, B) \leq C \Longleftrightarrow \varepsilon_{R}(C, B) \leq A .
$$

We prove this by the following series of transitions: $\delta_{R}(A, B)(y) \leq C(y) \forall y \in$ $X \Longleftrightarrow$

$\left(\bigvee_{x \in X}(R(x, y) * A(y)) \oplus B(y) \leq C(y) \forall y \in Y \Longleftrightarrow\right.$ (by Lemma 2) $(R(x, y) * A(y)) \oplus B(y) \leq C(y) \forall x, y \in X \Longleftrightarrow$ (by adjunction $(*, \oplus))$ $R(x, y) * A(y) \leq C(y) * B^{c}(y) \forall x, y \in X \Longleftrightarrow$ $A(y) \leq R(x, y) \mapsto\left(C(y) * B^{c}(y)\right) \forall x, y \in X \Longleftrightarrow$ (by Lemma 4) $\left.A(y) \leq(R(x, y) \mapsto C(y)) * B^{c}(y)\right) \forall x, y \in X \Longleftrightarrow$ $A(y) \leq \bigwedge_{x \in X}(R(x, y) \mapsto C(y)) * B^{c}(y) \forall x, \in X \Longleftrightarrow$ $A(y) \leq \varepsilon_{R}(C, B)(y) \forall y \in X$. 


\section{$6 \quad$ Fuzzy Morphological Spaces}

Basing on the concepts of stratified relational erosion and dilation and the results obtained in the previous sections, in this section we introduce the concept of a fuzzy relational morphological space and consider its basic properties. Special attention is made to interpreting these properties from topological point of view. To make exposition more homogeneous, in this section we assume that $(L, \leq$ $, \wedge, \vee, *)$ is a fixed quantale and operation $*: L \times L \rightarrow L$ distributes over arbitrary meets. Further, let $X$ be a set and $R: X \times X \rightarrow L$ be a reflexive symmetric $L$ fuzzy relation on the set $X$. Now, properties of the erosian operator obtained in in Proposition 3 and Corollaries 1, 2 and properties of dilation operator obtained established in Proposition 6 and Corollaries 3, 4 allow to get the following list of properties:

(1) $\varepsilon_{R}\left(a_{X}, B\right)=a_{X} * B^{c}$ for every $a \in L$.

(2) If $A_{1} \leq A_{2} \in L^{X}$ then $\varepsilon_{R}\left(A_{1}, B\right) \leq \varepsilon_{R}\left(A_{2}, B\right)$.

(3) Given $\left\{A_{i} \mid i \in I\right\} \subseteq L^{X}$, we have $\varepsilon_{R}\left(\bigwedge_{i \in I} A_{i}, B\right)=\bigwedge_{i \in I} \varepsilon_{R}\left(A_{i}, B\right)$.

(4) $\varepsilon_{R}(A, B) \leq A$ for every $A \in L^{X}$.

(5) $\varepsilon_{R}\left(\varepsilon_{R}(A, \bar{B}), B\right) \leq \varepsilon_{R}(A, B)$ for every $A \in L^{X}$

(5') $\varepsilon_{R}\left(\varepsilon_{R}\left(A, 0_{Y}\right), 0_{Y}\right)=\varepsilon_{R}\left(A, 0_{Y}\right)$ for every $A \in L^{X}$, and hence operator $\varepsilon_{R}\left(\cdot, 0_{X}\right)$ is idempotent.

(6) $\delta_{R}\left(a_{X}, B\right)=a_{Y} \oplus B$ for every $a \in L$.

(7) If $A_{1} \leq A_{2} \in L^{X}$ then $\delta_{R}\left(A_{1}, B\right) \leq \delta_{R}\left(A_{2}, B\right)$.

(8) Given $\left\{A_{i} \mid i \in I\right\} \subseteq L^{X}$, it holds $\delta_{R}\left(\bigvee_{i \in I} A, B\right)=\bigvee_{i \in I} \delta_{R}\left(A_{i}, B\right)$.

(9) $\delta_{R}(A, B) \geq A$ for every $A \in L^{X}$.

(10) $\delta_{R}\left(\delta_{R}(A, B), B\right) \geq \delta_{R}(A, B)$ for every $A \in L^{X}$.

(10') $\delta_{R}\left(\delta_{R}\left(A, 0_{Y}\right), 0_{Y}\right)=\delta_{R}\left(A, 0_{Y}\right)$ for every $A \in L^{X}$ and hence operator $\delta_{R}\left(\cdot, 0_{Y}\right)$ is idempotent.

Thus properties (1)-(5) remind basic properties of an $L$-fuzzy stratified preinterior Alexandroff operator int : $L^{X} \rightarrow L^{X}$ [2, Appendix A]. Moreover, in case when (5) is replaced by $\left(5^{\prime}\right)$, they are just the axioms of an $L$-fuzzy stratified interior Alexandroff operator. In its turn, properties (6)-(10) of the dilation operator $\varepsilon_{R}(\cdot, B)$ remind basic properties of the $L$-fuzzy stratified Alexandroff pre-closure operator $c l: L^{X} \rightarrow L^{X}[2$, Appendix A]. Moreover, in case when (10) is replaced by $\left(10^{\prime}\right)$, they are just the axioms of an $L$-fuzzy stratified closure Alexandroff operator.

Remark 1. Stratified interior means that int $\left(\alpha_{X}\right)=\alpha_{X}$ for all $\alpha \in L$ and stratified closure in our context mean that $\operatorname{cl}\left(\alpha_{X}\right)=\alpha_{X}$ for all $\alpha \in L$ (and not only for $\alpha=0$, see e.g. [17], [15]). The adjective Alexandroff means that the intersection axiom in the definition of interior and closure of an $L$-fuzzy topological space hold for arbitrary families (and not only finite) of (fuzzy) sets, see e.g. [1], [6]. Thus, the tuple $\left(X, R, \varepsilon_{R}, \delta_{R}\right)$ reminds the definition of a pre-di-topological space $[5]$.

Definition 11. A quadruple $\left(X, R, \varepsilon_{R}(\cdot, B), \delta_{R}(\cdot, B)\right)$ is called an L-fuzzy relational morphological space. 
Further, in case $B=0_{Y}$ operator $\varepsilon_{R}(\cdot, B)$ is idempotent by property $\left(5^{\prime}\right)$ and operator $\delta_{R}(\cdot, B)$ is idempotent by property $\left(10^{\prime}\right)$. Therefore by setting in an $L$-fuzzy relational morphological space $\left(X, R, \varepsilon_{R}(\cdot, B), \delta_{R}(\cdot, B)\right)$ families of $L$ fuzzy sets $\mathcal{T}_{R}=\left\{U \in L^{X} \mid \varepsilon_{R}(U, B)=U\right\}$ and $\mathcal{S}_{R}=\left\{V \in L^{X} \mid \delta_{R}(V, B)=V\right\}$, we obtain a fuzzy di-topological [5] space $\left(X, R, \mathcal{T}_{R}, \mathcal{S}_{R}\right)$. However, wishing to view it as a special type of an $L$-fuzzy relational morphological space, we call such spaces pure L-fuzzy relational morphological spaces - "pure" in the sense that they were not influenced by structuring.

To view $L$-fuzzy morphological spaces as a category $\mathbb{M}(L)$ and the category $\mathbb{M}^{p}(L)$ of pure $L$-fuzzy morphological spaces as its subcategory, we must specify its morphisms. We do it patterned after the topological background of these categories.

Definition 12. Let $\mathcal{X}_{1}=\left(X_{1}, R_{1}, \varepsilon_{R 1}, \delta_{R 1}\right)$ and $\mathcal{X}_{2}=\left(X_{2}, R_{2}, \varepsilon_{R 2}\right)$ be two $L$ fuzzy relational morphological spaces. A mapping $f: X_{1} \rightarrow X_{2}$ is called a continuous transformation from $\mathcal{X}_{1}$ to $\mathcal{X}_{2}$ if and only if the following conditions are satisfied:

1. $R_{1}(x, y) \leq R_{2}(f(x), f(y)) \forall x, y \in X_{1}$;

2. $f\left(\delta_{R_{1}}(A, B)\right) \leq \delta_{R_{2}}(f(A), f(B)) \forall A, B \in L^{X_{1}}$.

3. $f^{-1}\left(\varepsilon_{R_{2}}(A, B)\right) \leq \varepsilon_{R_{1}}(A, B) \forall A, B \in L^{X_{2}}$.

The proof of the following proposition is obvious

Proposition 9. Given three L-fuzzy morphological spaces $\mathcal{X}_{1}, \mathcal{X}_{2}$ and $\mathcal{X}_{3}$ and continuous transformations $f: \mathcal{X}_{1} \rightarrow \mathcal{X}_{2}$ and $g: \mathcal{X}_{2} \rightarrow \mathcal{X}_{3}$, the composition $g \circ f: \mathcal{X}_{1} \rightarrow \mathcal{X}_{3}$ is a continuous transformation. Given an L-fuzzy morphological space $\mathcal{X}$, the identity mapping $i d_{X}: X \rightarrow X$ is continuous.

Corollary 6. L-fuzzy morphological spaces and their continuous transformations constitute a category $\mathbb{M}(L)$.

Remark 2. In this section we did not assume any additional conditions on the quantale $(L, \leq, \wedge, \vee, *)$ except of the conditions supposed throughout the paper and meet-semicontinuity of the operation $*$. However, in case when $(L, \leq, \wedge, \vee, *)$ is a Girard quantale and/or satisfied conditions assumed in Theorem 3, some additional results, in particular, of categorical nature, can be obtained for the $L$-fuzzy relational morphological spaces. However, this will be the subject of the subsequent work.

\section{Conclusion}

In this paper, we have introduced the structured versions of $L$-fuzzy relational erosion and dilation operators defined on the $L$-power-set $L^{X}$ of the relational set $(X, R)$, generalizing (unstructured) $L$-fuzzy relational erosion and dilation counterparts introduced in [19] and further studied in [23]. After considering first separately and independently properties of $L$-fuzzy relational erosion and 
dilation we proceed with the study of interrelations between these operators. When developing the research in this direction we assume that $L$ is a Girard quantale and in some cases impose additional conditions on the operation $*$ in the quantale $L$. The main result here is that under assumption of some conditions the operators $\varepsilon_{R}$ and $\delta_{R}$ are dual and represent an adjunctive pair. In the last, fifth section we introduce category of $L$-fuzzy morphological spaces. Introducing these categories, we base on a certain analogy on behavior of erosion and dilation operators with topological operators of interior and closure.

As the main directions for the further research of structured $L$-fuzzy relational erosion and dilation operators and the corresponding categories of $L$-fuzzy morphological spaces we view the following.

- When studying the interrelations between structured $L$-fuzzy relational erosion and structured $L$-fuzzy relational dilation we had to impose some additional conditions on the quantale $L$, see e.g. Definitions 1 and 2. These conditions are sufficient but we do not know yet whether they are necessary. Probably these results can be obtained for some weaker conditions.

- In this paper, we address to the basic concepts of structured $L$-fuzzy relational mathematical morphology, namely erosion and dilation. Aiming to develop more or less full-bodied version of structured $L$-fuzzy relational mathematical morphology, as the second step we see the study of structured $L$-fuzzy relational opening and closing operators. At present we are working in this direction.

- As a challenging direction for the further research we consider the study of structured $L$-fuzzy relational morphological spaces, in particular to develop the categorical approach to $L$-fuzzy relational spaces.

- Quite interesting, especially from the point of possible application, will be to compare structured $L$-fuzzy relational morphological spaces with some kind of fuzzy rough approximation systems (cf $[9,10,20]$, etc,). In particular, it could be useful in the study of big volumes of transformed data.

- One of the main directions of mathematical morphology is image processing. Probably, also our approach will have useful application in this area.

Acknowledgement. The authors express appreciation to the anonymous referees for reading the paper carefully and making useful criticisms.

\section{References}

1. Alexandroff, P.: Diskrete Räume. Sbornik 2, 501-518 (1937)

2. Stadler, B.M.R., Stadler, P.F., Shpak, M., Wagner, G.P.: Recombination spaces, metrics and pretopologies. www.tbi.univie.ac.at/papers/Abstracts/01-02-011.pdf

3. Bloch, I.: Duality vs. adjunction for fuzzy mathematical morphology. Fuzzy Sets Syst. 160, 1858-1867 (2009)

4. Bloch, I., Maitre, H.: Fuzzy mathematical morphology: a comparative study. Pattern Recogn. 28, 1341-1387 (1995)

5. Brown, L.M., Ertürk, R., Dost, Ş.: Ditopological texture spaces and fuzzy topology, I. Basic concepts. Fuzzy Sets Syst. 147, 171-199 (2004) 
6. Chen, P., Zhang, D.: Alexandroff $L$-cotopological spaces. Fuzzy Sets Syst. 161, 2505-2525 (2010)

7. De Baets, B., Kerre, E.E., Gupta, M.: The fundamentals of fuzzy mathematical morphology part I: basic concepts. Int. J. Gen. Syst. 23, 155-171 (1995)

8. De Baets, B., Kerre, E.E., Gupta, M.: The fundamentals of fuzzy mathematical morphology part II: idempotence, convexity and decomposition. Int. J. Gen. Syst. 23, 307-322 (1995)

9. Dubois, D., Prade, H.: Rough fuzzy sets and fuzzy rough sets. Int. J. Gen. Syst. 17, 191-209 (1990)

10. Eļkins, A., Šostak, A., Uljjane, I.: On a category of extensional fuzzy rough approximation operators. Commun. Comput. Inform. Sci. 611, 36-47 (2016)

11. Gierz, G., Hoffman, K.H., Keimel, K., Lawson, J.D., Mislove, M.W., Scott, D.S.: Continuous Lattices and Domains. Cambridge University Press, Cambridge (2003)

12. Heijcmans, H.J.A.M., Ronse, C.: The algebraic basis of mathematical morphology: dilations and erosions. Vis. Gr. Image Process 50(3), 245-295 (1990)

13. Höhle, U.: M-valued sets and sheaves over integral commutative cl-monoids, Chapter 2. In: Rodabaugh, S.E., Klement, E.P., Höhle, U. (eds.) Applications of Category Theory to Fuzzy Subsets, pp. 33-73. Kluwer Academic Publishing (1992)

14. Höhle, U.: Many Valued Topology and its Application. Kluwer Academic Publisher, Boston (2001)

15. Höhle, U., Šostak, A.: Axiomatics for fixed-based fuzzy topologies, Chapter 3. In: Höhle, U., Rodabaugh, S.E. (eds.) Mathematics of Fuzzy Sets: Logic, Topology and Measure Theory - Handbook Series, vol. 3, pp. 123-272. Kluwer Academic Publisher (1999)

16. Jenei, S.: Structure of Girard monoid on [0,1], Chapter 10. In: Rodabaugh, S.E., Klement, E.P. (eds.) Topological and Algebraic Structures in Fuzzy Sets, pp. 277308. Kluwer Academic Publishing, Boston (2003)

17. Liu, Y.M., Luo, M.K.: Fuzzy Topology. Advances in Fuzzy Systems - Applications and Topology. World Scientific. Singapore (1997)

18. Matheron, G.: Random Sets and Integral Geometry. Wiley, New York (1975)

19. Madrid, N., Ojeda-Aciego, M., Medina, J., Perfilieva, I.: $L$-fuzzy relational mathematical morphology based on adjoint triples. Inf. Sci. 474, 75-89 (2019)

20. Pawlak, Z.: Rough sets. Int. J. Comput. Inf. Sci. 11, 341-356 (1982)

21. Rosenthal, K.I.: Quantales and Their Applications. Pirman Research Notes in Mathematics 234. Longman Scientific \& Technical (1990)

22. Serra, J.: Image Analysis and Mathematical Morphology. Academic Press, London (1982)

23. Šostak, A., Uljane, I.: Some remarks on topological structure in the context of fuzzy relational mathematical morphology. Atlantis Series in Uncertainty Modelling, vol. 1, pp. 776-783 (2019). https://doi.org/10.2991/eusflat-19.2019.106

24. Valverde, L.: On the structure of $F$-indistinguishability operators. Fuzzy Sets Syst. 17, 313-328 (1985)

25. Zadeh, L.A.: Similarity relations and fuzzy orderings. Inf. Sci. 3, 177-200 (1971) 